\title{
Coproduire la performance socialement responsable des collectivités territoriales : résultats émergents d'une recherche-intervention au sein d'une mairie
}

\author{
- Laurent Cappellettia ${ }^{a^{*}}$ et Samia Khenniche ${ }^{b}$ \\ ${ }^{a}$ Conservatoire National des Arts et Métiers, Chaire CCG, \\ 40 rue des Jeuneurs, 75002 Paris \\ ${ }^{b}$ Conservatoire National des Arts et Métiers, LIRSA-ISEOR
}

\section{Résumé}

L'article étudie les conditions du développement coproduit de la performance socialement responsable d'une collectivité territoriale. Pour cela, il mobilise une recherche-intervention menée au sein d'une mairie de 900 agents d'une commune de 50000 habitants. Les résultats émergents de la recherche montre que la performance socialement responsable d'une collectivité territoriale demande le recours à une méthode de management adaptée permettant une représentation co-construite de la performance publique entre les sphères administrative et politique, son évaluation systématique et une réduction de la distance cognitive entre les acteurs publics pour stimuler la synchronisation et le pilotage des politiques et la coproduction des stratégies publiques décidées.

๑ 2017 IDMP/Lavoisier SAS. Tous droits réservés

Mots clés: Performance socialement responsable, analyse socio-économique, diagnostic, groupe de projet,

comité de pilotage, management public coproduit, coûts performances cachés.

\section{Abstract}

Co-producing the socially responsible performance of local and regional authorities: emerging results of an intervention-research within a town hall. The article studies the conditions of the co-produced development of the socially responsible performance for a local authority. To this end, it mobilizes an intervention-research carried out in a town hall of 900 agents of a commune of 50,000 inhabitants. Emerging research findings show that the socially responsible performance of a local authority requires mainly: the use of a suitable management method allowing a co-constructed representation of public performance between the administrative and political spheres; its evaluation; and a reduction in the cognitive distance between public actors to stimulate both the synchronization and the steering of policies and the co-production of strategies.

(c) 2017 IDMP/Lavoisier SAS. Tous droits réservés

\footnotetext{
*Auteur correspondant : laurent.cappelletti@lecnam.net
}

doi :10.3166/pmp.34. 2017.0002 @ 2017 IDMP/Lavoisier SAS. Tous droits réservés 
Keywords: socially responsible performance, socio-economic analysis, diagnosis, project group, steering committee, co-produced public management, hidden costs.

\section{Introduction}

Depuis quelques années maintenant, s'observe une mutation profonde du contexte dans lequel les collectivités territoriales évoluent et portent le service public et qui réinterroge le rôle de l'agent public. En effet, la relation usager-collectivité devient plus directe et induit une exigence plus grande d'Accountability (Pettigrew, 1997), que l'on peut traduire par le terme « redevabilité ». Sur le plan institutionnel, les lois MAPTAM ${ }^{2}$ et $\mathrm{NOTRe}^{3}$ de 2015 ont provoqué de nombreux changements, parmi lesquels le développement des intercommunalités, la création des métropoles, et plus récemment encore la fusion des Régions. Elles impliquent des problématiques de répartition et de mutualisation des rôles, des missions, des ressources. Au niveau économique, la faible croissance économique et la crise de l'endettement des États et des collectivités impactent les ressources des collectivités du fait de la diminution des dotations de l'État (Cappelletti, 2016). Les collectivités doivent alors faire face dans un même temps à une nécessaire réduction des dépenses et un besoin d'accompagnement accru des populations exposées alors que la crise de 2007-2008 a renforcé la pression sur les attendus en termes de modernisation de l'action publique (Bartoli, Blatrix, 2015). Le développement des usages numériques vient appuyer des transformations démocratiques. Sur un temps long, ces évolutions s'inscrivent dans une tendance de fond relative à une transformation de ce qui fonde la légitimité de l'organisation publique. Au XIX ${ }^{\mathrm{e}}$ siècle il s'agissait du pouvoir, au $\mathrm{XX}^{\mathrm{e}}$ siècle la légitimité a reposé sur la finalité de l'organisation publique : le service public (Laufer, Burlaud, 1980). Aujourd'hui, ce qui semble primer est davantage la redevabilité. La légitimité est cette fois gagnée par la démonstration du sens de l'action et de la qualité de la conduite qui en est faite.

C'est pourquoi, depuis près d'une quarantaine d'années des expérimentations de gestion par résultats et de direction par les objectifs se font jour dans le secteur public, notamment sous l'impulsion du nouveau management public (New Public Management). Ces modes de gestion par résultats visent une gestion publique plus efficace, en favorisant la responsabilisation des équipes de gestion, la motivation des employés du secteur public et l'autonomie des managers à tous les niveaux organisationnels, le tout autour de la valorisation des prestations fournies aux citoyens (Mazouz et Leclerc, 2008). Si ces expérimentations se généralisent avec difficultés, le contexte actuel offre en retour une opportunité réelle de changement en raison des mutations institutionnelles, économiques et démocratiques qui le caractérise. Ces éléments conduisent les collectivités à s'interroger sur l'effectivité, l'efficacité et l'efficience, à travers la question de leur performance. Développer une performance durable de l'action publique, que nous qualifierons dans l'article de performance socialement responsable, suppose de considérer les dimensions internes - financière, organisationnelle, $\mathrm{RH}$ - et externes - de service public et territoriale. Il s'agit de développer une performance

\footnotetext{
${ }^{2}$ MAPTAM : modernisation de l'action publique territoriale et d'affirmation des métropoles

${ }^{3}$ NOTRe : nouvelle organisation territoriale de la république
} 
en phase avec les mutations institutionnelles, économiques et démocratiques, tout en respectant les exigences en termes de service public et d'animation territoriale en posant l'hypothèse que les collectivités disposent en leur sein des ressources nécessaires à cette transformation exploitables au travers des démarches s'appuyant sur la co-construction et la coproduction d'actions. L'une des grandes problématiques de gestion posée aux politiques publiques en France devient alors celle de la mesure des gains d'efficience demandés aux administrations et aux collectivités locales autant que la pertinence de ces gains eux-mêmes.

Finalement, à l'heure où les dotations de l'État vers les collectivités locales sont programmées pour diminuer de 11 milliards d'euros d'ici 2017 et de 13 nouveaux milliards d'ici 2022, les questions posées aux décideurs politiques des collectivités sont les suivantes. D'une part, où trouver les économies à réaliser pour gagner en efficience et absorber les baisses de dotations mais cela sans dégrader la qualité de la production de services publics ni la rétrécir dangereusement en désorientant l'usager? D'autre part, comment ne pas «braquer » les agents publics dans ces démarches de recherche d'efficience voire comment les impliquer dans l'action? Enfin, comment mesurer les économies et les gains d'efficience réalisés de façon fiable et communicable? Dans ce cadre, la problématique traitée dans l'article peut s'exprimer de la façon suivante : comment les collectivités peuvent développer une performance durable en phase avec les exigences contemporaines de l'action publique? $\mathrm{Ou}$ autrement dit, comment développer la performance des collectivités territoriales de façon socialement responsable, c'est-à-dire en trouvant une équilibration au sens de Perroux (1974) entre contraintes économiques et satisfaction sociale des agents et des citoyens-usagers? Pour apporter des éléments de réponse à cette problématique, l'article est organisé en trois parties. Dans la première, il explique comment les théories de la gestion publique se sont déplacées d'une approche de la performance publique univoquement comptable vers une approche plus socialement responsable appelant des démarches de co-construction et de coproduction d'actions (\&1). Puis au travers l'étude du cas d'une mairie de 900 agents d'une commune de 50000 habitants construite à partir d'une recherche-intervention, l'article identifie des premiers freins au développement de la performance socialement responsable d'une collectivité territoriale et les solutions pour y faire face notamment la co-construction et la coproduction des performances. Enfin l'article explicite les résultats observés quant aux conditions pour qu'une collectivité territoriale co-construise et coproduise sa performance socialement responsable, puis les discute (\&3).

\section{La performance de l'action publique : d'une approche comptable à une ap- proche coproduite plus socialement supportable}

L'analyse dans le temps des théories de la performance publique indique que celles-ci se sont déplacées d'une vision comptable de la performance, assez semblable à celle dominante dans le secteur privé, à une vision plus socialement responsable tendant à équilibrer l'économique, le social et le sociétal par la co-construction et la coproduction d'actions. Le cadre conceptuel présenté dans cette partie est nécessairement orienté, au regard de la problématique de l'article, vers les théories centrées sur la mise en œuvre des politiques publiques de façon performante et socialement responsable. C'est pourquoi il laisse de côté, sans nier l'intérêt de ces travaux en management public, des théories sociologiques 
assez éloignées des questions de mise en œuvre managériale comme celles de Boltanski et Thevenot (1991) ou encore celles plus tournées vers l'intégration des citoyens dans la définition des politiques publiques comme le proposent Ferlie, Hartley et Martin (2003) et moins vers la coopération des élus et des agents publics pour coproduire effectivement les politiques publiques au service des citoyens.

\subsection{Performance, efficacité et efficience de l'action publique : éléments théoriques}

Gibert (1980) dégage deux fonctions de production de l'action publique. La fonction interne correspond à la mise en œuvre de moyens dans la réalisation d'actions. La fonction externe est quant à elle relative aux impacts de l'action publique sur l'environnement de la collectivité. S'ajoute à la nécessaire efficacité de l'action publique, une « injonction » d'efficience. Ainsi, la notion de performance publique s'est répandue dans les discours des acteurs publics. Il s'agit d'orienter la gestion publique vers les résultats de son action et non plus seulement sur ses moyens. Carassus et al.(2011), à partir d'une revue de littérature des modèles contemporains de pilotage de la performance publique, proposent la définition suivante de la performance publique dans laquelle l'article s'inscrit : « la performance publique correspond à la capacité d'une organisation publique à maîtriser ses ressources humaines, financières et organisationnelles, afin de produire une offre de services publics adaptée, en qualité et quantité, répondant aux besoins de ses parties prenantes et générant des effets durables vis-à-vis de son territoire ». On est bien parvenu aujourd'hui, en théorie, avec cette définition à une vision de la performance publique socialement responsable c'est-à-dire partagée entre ses différentes parties prenantes et agrégeant l'économique, le social et le sociétal.

Néanmoins, au niveau pratique des collectivités territoriales, les démarches visant à développer le niveau de performance requis par les conditions économiques tout en assurant un niveau acceptable de satisfaction sociale et sociétale demeurent rares (Carassus, Gardey, 2009; Trébucq, 2016). Pourquoi cela? Une des problématiques majeures relevée dans la littérature réside dans la difficulté d'accorder la notion de performance publique avec celle de service public fondé sur des principes d'éthique de redistribution, d'équité et de solidarité (Bartoli et al., 2011), et de fédérer des acteurs aux logiques multiples autour d'une stratégie commune de service public en phase avec les nouvelles attentes des usagers et partenaires. Si la thématique de la performance durable est désormais ancrée dans le champ du management public, elle peine ainsi à s'inscrire dans les pratiques. Dans le détail, la littérature a principalement cerné trois facteurs explicatifs de ces difficultés : les nécessaires et profonds changements de logiques institutionnelles impliqués, la délicate définition puis évaluation des résultats et enfin la gestion de la distance cognitive entre politique et administration. Tout d'abord, si les notions relatives à la performance se diffusent dans la sphère publique, elles demeurent empreintes d'une certaine connotation négative. Les dernières réformes de l'administration naissent dans un contexte politique où le rôle et le poids de l'action publique sont régulièrement questionnés. Inspirées par le Nouveau Management Public (New Public Management), ces réformes reposent volens nolens sur une doctrine économique classique qui peut sembler opposée aux valeurs traditionnelles du service public en développant une approche univoquement comptable et financière de la performance (Davis, West, 2009; Bartoli et al., 2011). Néanmoins, comme le souligne Favoreu et al. (2015), depuis quelques années, on observe une évolution d'une conception 
«statique » de la performance, centrée sur la mesure et l'évaluation comptable, vers une conception plus systémique et holistique (Moynihan, 2008) s'intégrant ainsi dans la prise de décision budgétaire (Moynihan, 2006), la gestion des ressources humaines (Den Hartog et al., 2004) et les politiques publiques (Bouckaert, Van Dooren, 2002). Dans le même temps, le constat général est que les démarches de performance et de modernisation menées selon le modèle de New Public Management ont davantage engendré de tensions et de crispations que de résultats tangibles en termes d'amélioration de la qualité de service public et de maitrise de l'affectation des fonds publics (Mazouz, Tremblay, 2006; Mazouz, Tardif, 2010). Un autre frein relevé par la littérature est la difficulté des gestionnaires publics à passer d'une logique d'administration publique à une logique de management public (Laufer et Burlaud, 1980). La faible capacité à dépasser la logique de bureaucratie administrative et à intégrer des formes d'organisation ouvertes aux partenaires externes, adaptées à l'apprentissage organisationnel, à la co-construction des projets et au co-pilotage (Mazouz et al., 2015) freine cette transition. La dimension instrumentale de la logique managériale témoigne également d'une transition : la gestion de la performance s'appuie sur des outils orientés vers les résultats, avec une logique d'efficience, elle diverge de la logique bureaucratique qui met l'accent sur les règles et les moyens (Laufer, Burlaud, 1980; Meyer et al., 2013). De plus, les recherches pointent également la difficile définition et évaluation des résultats attendus du secteur public, notamment en comparaison du secteur privé (Laufer, Burlaud, 1980 ; Trosa 1989). Concernant les collectivités locales, plusieurs éléments rendent l'exercice en effet délicat :

- la diversité des cibles de l'action publique aux attentes différentes voire contradictoires : contribuables, usagers, administrés, acteurs associatifs, économiques et institutionnels ;

- la répartition des compétences entre différentes autorités qui diminue la prise des collectivités sur leur territoire (Ring, Perry, 1985);

- la multiplicité du temps (Martinet, 1991) dans lesquels l'action publique est conduite : temps du projet, du mandat, des cycles économiques.

Devant une telle complexité, la mise en place d'objectifs de performance cohérents et d'indicateurs de pilotage pertinents, s'inscrivant dans une stratégie d'ensemble, s'avère malaisée. Ainsi, même lorsque les valeurs véhiculées par le management par la performance sont admises et acceptées, de nouveaux dissensus peuvent émerger et venir entraver la conception de la stratégie à adopter. En effet, si la gestion par les résultats s'inscrit dans un cadre déterminé, il ne s'agit pas d'une démarche mécanique (Martin, Jobin, 2004), mais bien d'une « transformation culturelle » qui ne peut donc incomber à la seule administration (Mazouz, Rochet, 2005). Un double portage politique et administratif est indispensable ce qui suppose pour les acteurs concernés de dépasser leur distance cognitive.

\subsection{Réduire la distance cognitive entre les acteurs publics}

Le troisième frein à la performance durable des collectivités territoriales mis en exergue par la littérature est celui de la distance cognitive entre les acteurs publics. Van Hée (2008) entend par distance cognitive la différence entre individus sur le plan des connaissances, mais également dans leur manière de percevoir et d'interpréter les phénomènes. Plus les schémas mentaux sont dissemblables, plus la distance cognitive qui les sépare est grande. Selon Nooteboom (2000), la capacité innovatrice de la collaboration entre deux acteurs repose 
d'une part sur la capacité d'absorption entre partenaires qui est fonction décroissante de la distance cognitive entre eux. Elle repose d'autre part sur la valeur de la nouveauté apportée par chaque partenaire qui est fonction croissante de la distance cognitive. Performance et innovation impliquent donc de gérer la distance cognitive entre acteurs pour limiter ses effets néfastes sur la capacité à coopérer efficacement et sur la coproduction de politiques publiques fondée sur des représentations co-construites. Notons que ce concept de distance cognitive utilisé dans l'article s'inscrit dans un cadre de gestion. Il est donc distant du sens qu'il pourrait avoir en cognition sociale. Il repose essentiellement, pour mieux le positionner, sur les concepts de rationalité limitée de Simon (1957) et d'apprentissage organisationnel d'Argyris et Schön (1978). Dans le cadre de la relation entre les élus et la direction générale d'une collectivité, un subtil jeu relationnel entre distance et proximité est observé (Robin, Desfontaines, 2015). Ce jeu repose sur une claire répartition des rôles à même de maintenir un lien sans confusion. Or, l'intégration d'une gestion par objectif interroge la place des gestionnaires publiques face aux élus et vient troubler cet équilibre politico-administratif (Mazouz, 2008; Facal, Mazouz, 2013). Côté administration, il est attendu une capacité à répondre rapidement, de manière souple et adaptée aux préoccupations des bénéficiaires de l'action publique. Cela requiert plus d'autonomie dans la décision, plus d'innovation dans les pratiques, et un certain « sens politique » (Maltais, Mazouz, 2004). Côté politique, leur plus grande visibilité de la gestion de l'activité leur offre la possibilité d'intervenir davantage dans les décisions de mise en œuvre de l'action. On observe une sorte de «managérialisation » du politique (Robert, 2007).

Or, la littérature en management public converge pour identifier une perte de confiance de l'administration ainsi que du politique lié en partie à une distance cognitive croissante entre les deux sphères quant à ce que devrait être la performance publique. Aussi, redonner confiance aux acteurs dans une institution comme une collectivité territoriale suppose que les fonctionnaires et les élus de cette collectivité retrouvent également confiance en euxmêmes puis coopèrent en s'accordant sur ce que devrait être leur performance mutuelle. Au fond c'est à une co-construction/coproduction de la mise en œuvre des politiques publiques qu'appelle la littérature pour retrouver la confiance des citoyens, des agents publics et des élus (Christensen, Laegreid, 2010 ; Ashworth et al., 2013 ; Meier et al., 2015). En pratique ce n'est pas le cas général aujourd'hui ou la qualité de gestion des collectivités territoriales fait l'objet de critiques foisonnantes aggravant la perte de confiance non seulement de ses usagers externes mais aussi celle de ses acteurs internes agents et élus (Cappelletti, 2016). Selon l'approche socio-économique du management public (Savall, 1974; Savall, Zardet, Bonnet, 2008; Noguera, 2006, 2008; Savall, Zardet, 2010; Zardet, Noguera, 2013 ; Cappelletti, 2016) il est possible de restaurer la confiance du fonctionnaire et de l'élu en réduisant leur distance cognitive par un management socio-économique jouant à la fois sur la satisfaction sociale et sociétale et sur une consommation plus efficiente des ressources. C'est du reste le sens ce qui a été mis en œuvre peu ou prou dans les administrations et les collectivités de pays tels que le Canada, la Suède, l'Allemagne, la Suisse voire l'Italie (Cappelletti, 2016). L'approche socio-économique du management public montre aussi qu'une collectivité territoriale dispose de réserves considérables d'efficacité convertibles en performances au travers de démarches participatives et structurées permettant la co-construction puis la coproduction d'actions. Ces réserves sont liées aux coûts engendrés par les défauts de coopération entre les sphères administratives et politiques qui se traduisent par des dysfonctionnements dans cinq 
domaines principaux au sein des collectivités territoriales : l'absentéisme, les accidents du travail et les maladies professionnelles, les défauts de mobilité et de rotation du personnel, les non-qualités de services publics et les écarts de productivité directe ou, autrement dit, les sous efficacités (Noguera, 2006, 2008; Savall, Zardet, 2010). Les études en la matière montrent qu'au bas mot la moitié de ces déperditions économiques est recyclable en performances et ressources nouvelles, l'autre moitié étant incompressible ou plus contrainte. Pour l'approche socio-économique, dans la plupart des organisations publiques - comme du reste dans les entreprises privées mais pour d'autres raisons - les déperditions économiques engendrées par des défauts d'organisation et de gestion sont ainsi très significatives de l'ordre de $20000 €$ jusqu'à $60000 €$ par personne et par an (Savall, Zardet, Bonnet, 2008; Cappelletti, 2012). Une fois mesurées et exploitées les ressources endogènes, l'approche socio-économique du management public indique en outre qu'elles peuvent être utilisées pour absorber les baisses de dotations de l'État, pour rétribuer en plus les fonctionnaires méritants et/ou pour financer de nouveaux programmes d'aide dans le territoire considéré et renouveler l'offre de services publics. Les trois facteurs clés d'une telle gestion seraient les suivants selon cette approche (Noguera, 2006, 2008; Savall, Zardet, 2010) :

- un engagement politique sans faille des élus des collectivités territoriales pour la mettre en œuvre;

- une méthodologie participative, structurée et négociée pour identifier dans toutes les équipes et les services des collectivités considérées les dysfonctionnements et mettre en œuvre des plans d'actions socialement supportables de conversion des coûts en performances. Une telle méthodologie reposant sur la co-construction puis la coproduction de la mise en œuvre des politiques publiques désirées ;

- une contractualisation pour reverser une fraction des gains réalisés aux agents des services ayant atteint les objectifs des plans d'actions et autofinancer des plans de carrière plus stimulants qui entretiennent la motivation des agents.

\subsection{La mesure des gisements endogènes d'efficience dans les collectivités territoriales}

Point intéressant dans la littérature en management public, l'approche socio-économique a identifié deux archétypes de dysfonctionnements générateurs de déperditions économiques au sein d'une collectivité territoriale. D'une part ceux qui résultent de décisions politiques de dépenses et qui vont être jugés comme ne contribuant pas (ou plus) à la production attendue de services publics (a). D'autre part, ceux engendrés par les dysfonctionnements d'organisation et qui engendrent des coûts le plus souvent cachés par les systèmes d'information traditionnels dont les budgets (b).

\section{Exemples de dépenses jugées dysfonctionnelles au plan politique}

Les exemples de dépenses publiques réalisées par une collectivité et qui pourraient être remises en cause par de nouvelles orientations politiques sont nombreux. Ils représentent un premier gisement conséquent de ressources économiques. Par exemple :

- achat d'un ordinateur pour tous les lycéens d'une région;

- saupoudrages de subventions qui partant deviennent inutiles.

Les coûts de ces décisions sont visibles dans le budget et leur réduction est « techniquement » aisée consistant à décider d'arrêter les dépenses considérées sur des critères politiques. 


\section{Exemples de déperditions économiques engendrées par des défauts d'organisation}

Les exemples de coûts cachés engendrés par les dysfonctionnements d'organisation et de gestion au sein d'une collectivité sont plus nombreux encore. Ils représentent la partie dominante des gisements de ressources internes exploitables par une collectivité territoriale. Parmi les exemples les plus «génériques », l'approche socio-économique a repéré par exemple (Cappelletti, 2012) :

- le coût de l'absentéisme lié à des défauts de conditions de travail et de management de proximité des agents;

- les heures supplémentaires compressibles liées à des défauts évitables d'organisation du travail;

- Des tâches faites en double au sein d'un service ou entre services, des arrêts de travail liés à des pannes informatiques, des surconsommations de biens ou services pour achever des productions de services publics (dossiers administratifs, manifestations publiques etc.) qui présentent des défauts de qualité.

À la différence des dysfonctionnements et des coûts qu'ils entraînent de type (a) qui vont demander des solutions politiques d'explication pour les réduire le cas échéant, les coûts cachés engendrés par les dysfonctionnements d'organisation de type (b) demandent une méthodologie adaptée pour les identifier puis les réduire au travers des solutions de transformation négociées entre l'administration et le politique et coproduites.

Ainsi, l'inscription de la performance durable dans les pratiques des collectivités ne peut être uniquement le fait d'un contexte économique, démocratique et institutionnel pressurisant. Une évolution interne, stratégique, est requise. À travers l'étude des premiers résultats d'une recherche-intervention en cours au sein d'une mairie, l'article va identifier les principales difficultés rencontrées par les acteurs publics pour mettre en œuvre une gestion de la performance socialement responsable, les solutions pour y faire face, et confronter ces premiers résultats avec la littérature en management public notamment l'approche socio-économique.

\section{Conception et protocole de la méthodologie de recherche-intervention menée au sein d'une mairie de taille moyenne}

Une mairie de 900 agents d'une commune de taille moyenne de 50000 habitants située en Ile-de-France a chargé sa direction générale des services (DGS) d'une mission pour développer en son sein une performance socialement responsable. Pour accompagner la DGS dans cette mission, la mairie a souhaité faire appel à une équipe de recherche-intervention pour, dans un premier temps (2015-2016), établir un diagnostic qualitatif co-construit des freins à la mise en œuvre de la performance durable et coproduire des premières solutions pour les réduire. Puis dans un deuxième temps (2016-2017), évaluer économiquement les coûts des dysfonctionnements d'organisation et de coopération et les performances engendrées par les solutions coproduites pour les traiter. C'est notre équipe de recherche qui a été retenue (un chercheur immergé au sein de la collectivité territoriale pour mettre en œuvre le protocole de recherche et collecter les données en binôme avec un chercheur distancé pour contrôler les données, les exploiter et les positionner) par la mairie au travers un appel d'offres complété par une série de réunions avec les décisionnaires de la mission : le maire, son directeur de cabinet, le directeur général des services, la DRH, l'adjoint aux finances. Ce sont les résultats de la première phase de la recherche menée de 2015 à 2016 qui sont présentées dans l'article. 


\subsection{Le design de la recherche-intervention mobilisée pour traiter la problématique soulevée}

La méthodologie de recherche-intervention est apparue comme bien adaptée au traitement de la problématique de l'article qui nécessitait des observations longitudinales de terrain réalisées au travers des interactions avec les acteurs concernés et cela dans un contexte transformatif. La recherche-action, qui s'incarne dans la célèbre formule de Lewin «One of the best way to know the world is to change it » (cité par Argyris et al., 1985), est la forme la plus connue de recherche transformative (Jönsson, Lukka, 2005). Et incontestablement, la recherche-intervention présente des convergences avec la recherche-action. Mais elle s'en distingue sur un point fondamental concernant la conception que chacune des deux méthodologies se fait de la transformation. En effet la recherche-action, dans sa forme canonique, est plus tournée vers la formalisation du changement et moins vers sa contextualisation (David, 1999; Reason, Bradbury, 2001). La recherche-intervention vise quant à elle la formalisation ET la contextualisation du changement. Elle cherche à transformer effectivement l'organisation dans ses structures et ses comportements, et non pas seulement à préparer/décrire des changements futurs. Cette différence explique peut-être pourquoi la recherche-action est tombée quelque peu en désuétude dans les recherches en management et gestion, alors que la rechercheintervention s'est répandue au plan scientifique et académique, singulièrement dans les pays anglo-saxons et scandinaves, par exemple au travers les travaux très connus en Organizational Development (OD) et Management Control (MC) de Chris Argyris, Donald Schön, Edgar Schein, Jeffrey Pfeffer, Robert Kaplan, Warren Bennis ou Karri Lukka (Cappelletti, 2007).

La recherche-intervention menée dans la présente étude a consisté en premier lieu à expérimenter sur un terrain d'observation une transformation - la mise en œuvre d'un management de la performance socialement responsable en l'occurrence - pour, dans une optique technique, améliorer la gestion et le management du terrain, et, dans une optique scientifique, faire des observations en vue d'identifier des régularités et des spécificités et de les positionner dans le champ théorique en vigueur. D'autre part, l'expérimentation présentée a été faite selon les phases de la boucle de recherche qui caractérise la recherche-intervention. Ces phases forment un processus itératif qui vise à l'élaboration de théories à partir des pratiques, par accumulation d'observations validées (David, 2003, 2004). Elles consistent à formuler les hypothèses de la recherche, à construire et mettre en œuvre un dispositif intégré d'observations, puis à discuter les résultats et toiletter les hypothèses en perspective d'une prochaine recherche. Il y a donc une alternance des travaux de terrain (phase d'immersion) avec des phases de recul et d'analyse de ces travaux (phase de distanciation) qui confère au processus de recherche une dimension logico-inductive et hypothético-déductive (Burrel, Morgan, 1985; Mckelvey, 2006). Ces phases de la rechercheintervention reposent sur une épistémologie intégrée fondée sur trois principes principaux (Van de Ven, Johnson, 2006; Buono, Savall, 2007). Le premier est celui dit « d'interactivité cognitive » qui signifie que les connaissances sont coproduites par le chercheur et les acteurs lors de dispositifs interactifs, tels que par exemple des diagnostics et des groupes de projet. Le second est celui dit «d'intersubjectivité contradictoire » qui signifie que les observations sont validées par discussions et débats entre le chercheur et les acteurs, par exemple lors de séances de groupes de projet ou de comité de pilotage. Enfin, le troisième est celui dit « de contingence générique » qui consiste au travers un retour vers la littérature, des études de cas passées et la communication des résultats de la recherche à identifier les connaissances contingentes (locales) de celles génériques (reproductibles) tirées de la recherche (Wacheux, 1996; Maxwell, 1996; Cappelletti, 2007). 


\subsection{Le protocole de la recherche-intervention au sein de collectivité territoriale étudiée}

Le projet de recherche-intervention a été élaboré de concert avec les principaux décideurs politiques et administratifs de la ville. Il correspondait à une demande de la mairie étudiée de l'aider à développer concrètement sa performance de façon socialement responsable. Ont été impliqués dans le processus des membres de l'administration - directeur général des services en charge des questions de performance publique, directeur des ressources humaines - et de l'exécutif - maire, adjoint aux finances et RH, directeur de cabinet. Le protocole final est le fruit de nombreuses concertations avec ces acteurs. L'implication des différentes parties composant de la gouvernance de la collectivité a paru essentielle au processus de transformation nécessaire à l'émergence d'une représentation partagée de la notion de performance durable de l'action publique.

Dans le détail, les travaux porte sur une municipalité d'Ile-de-France de taille moyenne (50000 habitants). Ancienne ville nouvelle, elle a connu un accroissement démographique rapide (7000 habitants en 1970), accompagné d'un développement soutenu en termes d'aménagement du territoire et d'organisation de la collectivité et des services publics. La population se caractérise par sa jeunesse, des revenus relativement faibles, et un besoin fort d'accès aux services publics. Située dans la grande couronne parisienne, la municipalité connaît actuellement une mutation institutionnelle profonde liée à la loi de modernisation de l'action publique territoriale et affirmation des métropoles (MAPTAM) et à la création d'une nouvelle intercommunalité de plus de 200000 habitants. L'histoire récente de la collectivité est également marquée par la crise financière de 2008 , qui a constitué un tournant en termes d'attendus de la gestion publique. Compte tenu des contraintes financières fortes, la logique d'efficacité ne peut plus être découplée de celle d'efficience. Le contexte économique influence également les besoins de services publics : la population du territoire particulièrement impactée en termes de perte d'emplois et de ressources financières accroît ses demandes de services publics. C'est dans ce contexte que se dessine la volonté d'orienter la gestion de la ville vers une logique de résultats socio-économiques, en adéquation avec l'évolution du territoire et les besoins de la population, tout en assainissant les dépenses publiques. La question de la performance publique socialement responsable est ainsi inscrite dans le programme municipal de l'équipe en place et une direction dédiée est créée. Un an et demi après le début de la recherche-intervention, sa première phase est achevée. Quelques transformations ont eu lieu mais à ce stade aucune stratégie ou feuille de route n'a pu être encore stabilisée ce qui illustre le difficile chemin de l'intention à l'action sur un sujet telle que la performance durable dans une collectivité territoriale. Le premier dispositif de la recherche présentée dans l'article s'est déroulé de septembre à octobre 2015. Il a consisté en la réalisation d'un diagnostic qualitatif qui a permis de faire expliciter aux différents acteurs leur appréhension de la performance de l'action publique, ainsi que de dégager les obstacles à son développement. Son second dispositif, un groupe de projet, a permis d'accompagner les acteurs dans la définition des leviers de développement de la performance durable de leur collectivité. Cette seconde séquence est intervenue d'octobre 2015 à mars 2016. Au-delà du diagnostic et du groupe de projet, des réunions de pilotage avec la direction générale des services et le maire, et la tenue d'un journal de bord lors des nombreux échanges avec les acteurs ont permis également de collecter de nombreuses observations complémentaires. 


\section{La technique de diagnostic utilisée}

Le diagnostic a été élaboré sur la base d'entretiens semi-directifs conduits auprès des membres de l'exécutif et de la direction générale de la mairie étudiée afin de déterminer les différentes représentations de la performance publique et d'identifier ses limites. Le guide d'entretien (tableau 1) a été élaboré à partir d'une revue de littérature en management public, en mobilisant la grille de lecture de Carassus et al. (2011) qui caractérise la performance publique par ses dimensions internes et externes et qui rejoint donc une conception de la performance socialement responsable (Boukaert, Pollitt, 2004).

Tableau 1 : Thèmes et sous-thèmes de la grille d'entretien

\begin{tabular}{|l|l|}
\hline Thèmes & Sous-thèmes \\
\hline $\begin{array}{l}\text { 1- Dimension interne de la performance } \\
\text { publique }\end{array}$ & $\begin{array}{l}\text { 1-1- Organisation } \\
1-2-\text { Finance }\end{array}$ \\
& 1-3- Ressources humaines \\
\hline $\begin{array}{l}\text { 2- Dimension externe de la performance } \\
\text { publique }\end{array}$ & $\begin{array}{l}\text { 2-1- Service public } \\
\text { 2-2-Territoire }\end{array}$ \\
\hline
\end{tabular}

21 entretiens semi-directifs d'une heure ont été conduits :

- 14 auprès des membres de l'exécutif : maire, 11 adjoints au maire, directeur et chef de cabinet;

- 7 auprès des membres de la direction générale : directeur général des services, et directeurs généraux adjoints.

Ces entretiens ont consisté à interroger les acteurs sur les limites vécues ou observées concernant la performance durable de l'action publique de la collectivité. Le verbatim collecté a été traité manuellement, puis structuré en arborescence. 272 extraits ont été classés en 5 thèmes, 13 sous-thèmes. Cette technique de diagnostic qui vise donc à la co-construction des situations de gestion offre également l'opportunité d'analyser les convergences et les spécificités entre les représentations de la performance publique des élus et celles de la direction générale.

\section{La technique de groupe de projet utilisée}

Le groupe de projet stratégique a réuni l'ensemble des membres de l'exécutif et de la direction générale ayant participé au diagnostic. Sur la base d'une synthèse du diagnostic, des axes de travail ont été définis afin de concevoir des actions de transformation et des leviers de développement d'une performance socialement responsable. Quatre séances collectives ont eu lieu. La première séance a permis d'exposer la synthèse hiérarchisée du diagnostic. Cette synthèse du verbatim, formulée par les chercheurs sur la base de leurs observations réalisées in situ et d'éléments de connaissance génériques relatifs aux organisations publiques, a fait l'objet d'échanges entre les acteurs de l'organisation, et entre ces acteurs et les chercheurs. De ces échanges sont issus les axes de transformation prioritaires, démultipliés en idées-forces, pour chacune desquelles un binôme politicoadministratif a été formé. À travers ces binômes, il s'est agi de favoriser la coopération entre politique et administration afin de dépasser les tensions de gouvernance identifiées au sein de la collectivité. Au total, 18 binômes ont été formés. Chacun des binômes s'est 
ensuite réuni dans l'intervalle des séances collectives une et deux, puis deux et trois, afin de concevoir des actions de développement de la performance socialement responsable. Les séances collectives deux et trois ont permis la présentation des travaux des binômes. Les objectifs et les actions prioritaires présentés ont fait l'objet d'arbitrage, de hiérarchisation, de priorisation. À travers ces discussions, un rapprochement cognitif entre les membres de l'administration et les élus a pu être observé. La dernière séance (séance quatre) a donné lieu à des ajustements et à la validation du plan d'actions stratégiques formalisé. Si tous les objectifs et actions n'ont pu être passés au crible de manière collégiale, elles ont néanmoins fait l'objet d'une validation par le directeur général des services pour l'administration, et par une équipe resserrée d'élus pour l'exécutif. Cette dernière séance a par ailleurs permis un échange approfondi entre élus, et entre élus et administration, autour d'une des décisions phare du groupe de projet, la reconfiguration des pôles de politiques publiques (voir \&3). Cette méthodologie de groupe de projet vise à la coproduction de la mise en œuvre des politiques publiques à l'échelle d'une collectivité entre élus, agents et chercheurs.

L'animation des séances de restitution de diagnostic et de groupe de projet ont été conduites par les chercheurs selon les principes d'interactivité cognitive et d'intersubjectivité contradictoire propre à la recherche-intervention, afin de favoriser l'émergence d'éléments de connaissances, mais également de stabiliser un consensus opératoire entre les acteurs de la collectivité; consensus propice à la transformation durable de l'organisation. Lors des nombreux échanges qui ont ponctué les différentes étapes de la recherche-intervention, des éléments de connaissance de management public ont pu être vérifiés (régularités ou données génériques), alors que d'autres éléments ont émergé (spécificités ou données contingentes).

\section{Des résultats qui esquissent les conditions du développement coproduit de la performance durable d'une collectivité territoriale}

Comme exposé préalablement, développer une performance socialement responsable, suppose de considérer à la fois les dimensions internes - financière, organisationnelle, $\mathrm{RH}$ - et externes - de service public et territorial, en intégrant les mutations institutionnelles, économiques et démocratiques, et en respectant les exigences en termes de service public et d'animation territoriale.

\subsection{L'identification de trois freins principaux au développement de la performance durable}

Un premier niveau d'analyse des résultats du diagnostic révèle la faible expression relative à la dimension externe de la performance. Cette dernière totalise un peu plus d'un tiers de l'expression, mais ce score est à relativiser par l'explicitation d'un biais d'actualité : les entretiens ont été réalisés alors que la nouvelle intercommunalité, issue de la loi MAPTAM, était en pleine création, générant craintes et mécontentements chez les élus et les membres de la direction générale. Ainsi, sur les $34 \%$ d'expression relative à la dimension externe, $70 \%$ concernent les relations à l'intercommunalité actuelle en place ou celle à venir (soit $25 \%$ de l'expression totale). Les autres partenaires de la collectivité (Département, État, Associations, notamment), n'ont quant à eux recueilli que très peu d'expression de la part des acteurs. La question des besoins et impacts de l'animation territoriale a été quant à elle faiblement abordée (mois de $4 \%$ d'expression au total). Autre résultat marquant relatif à la dimension externe : la 
faible part d'expression concernant le thème Service public, correspondant à $8 \%$ de l'expression totale. Parmi les items recueillant une part d'expression particulièrement faible, notons celui des besoins et impacts des services publics. Ces résultats mettent en évidence un contraste entre l'approche durable de la performance de l'action publique, qui requiert de considérer l'exigence de qualité de service public et d'animation territoriale, et l'approche des acteurs de la collectivité en début de recherche-intervention. L'attention de ces derniers se porte principalement sur la dimension interne de la performance, alors même que l'action publique se caractérise par sa dimension externe, à savoir les impacts des politiques menées sur les populations et territoires.

Un second niveau d'analyse et de mise en perspective de ce verbatim à la lumière des observations réalisées in situ contribuent à expliquer ce contraste entre l'expression des acteurs et leur volonté de développer une performance socialement responsable. Trois axes se détachent : le manque de clarté de la définition politique et stratégique, la surpondération de la dimension financière et enfin les tensions de rôles entre politique et administration. Ces trois problématiques, particulièrement prégnantes dans la collectivité, apparaissent comme les premiers obstacles à transcender pour transformer l'intention de performance socialement responsable en action effective.

\section{Le manque de clarté de la définition politique et stratégique}

L'expression des acteurs se concentre non pas sur la dimension instrumentale de la performance de l'action publique, mais bien en amont, dans la définition même de la portée et du sens assignés à cette performance (voir encadré 1).

\section{Encadré 1 - Expression relative à la définition stratégique (extrait)}

«Il faut se poser la question de ce sur quoi doit se concentrer l'action publique. On se pose souvent cette question sous la contrainte. Tant qu' on est dans le discours ça va, quand il faut passer à l'acte, chacun veut garder son système. » (Exécutif)

« Notre service public répond bien aujourd' hui, mais demain? On a des quartiers en renouvellement urbain; au nom de quoi faudrait-il reproduire les mêmes équipements? On s'est parfois cassé les dents sur le dos des élus sur ce genre de questions, donc on est un peu refroidis. » $(D G)$

Si l'impératif de performance est bien entendu, deux difficultés majeures se font jour. La première concerne l'intégration de la performance socialement responsable dans le projet politique. Lorsque les acteurs ont été interrogés sur la définition stratégique de la performance de l'action publique, ils se sont finalement exprimés sur le projet politique qui sous-tend cette définition stratégique pour faire part de leur difficulté à réinterroger le projet politique à l'aune de cette exigence de performance. La deuxième difficulté réside dans la nécessité de transformer les pratiques dans son propre périmètre, dans sa propre délégation, et ce de manière durable. D'une part parce que la notion de performance collective renvoie les dirigeants publics à leur capacité à mener l'action publique, et suppose une remise en question. D'autre part parce que lorsque la prise de conscience a lieu, reste la difficulté à conduire le changement.

Lorsque l'accord sur les lignes politiques est établi, reste la difficulté de passer de la politique à la formalisation d'une stratégie, puis de la stratégie à la démultiplication stratégique (voir encadré 2). On retrouve dans l'expression des acteurs les idées de consensus 
« discouriel », relatif à l'intention, et de dissensus stratégique, relatif à une mise en action à visée structurante. La performance est alors portée dans le discours politique, mais ne se traduit pas en actes concrets, perceptibles par les usagers. Ainsi dans la municipalité étudiée, la question de la performance figurait dans le programme politique de campagne, puis dans le programme de l'équipe municipale. Sa traduction dans l'administration s'est concrétisée par la création d'une direction dédiée. Malgré l'intention politique et le portage politico-administratif, la mise en ouvre n'a pas eu lieu. Dix-huit mois se seront écoulés entre la création des services dédiés et la formalisation d'un premier plan stratégique relatif à la performance publique, conçu dans le cadre de la recherche-intervention.

\section{Encadré 2 - Expression relative à la démultiplication stratégique (extrait)}

"On a trop tendance à être flou dans les objectifs. Mettre des objectifs qualitatifs et quantitatifs c'est presque un gros mot.» (Exécutif)

"Il y a un fossé entre le discours politique et l'information donnée à la population. Le service public n'est pas porté que par une équipe de 30 élus, mais aussi par les agents sur le terrain. » (DG)

Un autre facteur qui vient limiter la mise en action d'une performance socialement responsable de la ville est la faiblesse des pratiques d'évaluation des politiques publiques (voir encadré 3). Cette faiblesse limite la capacité de réflexivité et de ré interrogation des politiques conduites à l'aune de leurs impacts sur les populations et territoires concernés.

\section{Encadré 3 - Expression relative aux pratiques d'évaluation des politiques publiques (extrait)}

«On va vite, on est beaucoup sollicités. On ne s'arrête pas assez pour s'évaluer, savoir ce qu'on a fait, comment on l'a fait. » (Exécutif)

«Ici c'est le dernier qui a parlé qui a raison, on ne mesure pas pour décider. »(DG)

\section{La surpondération de la dimension financière de la performance publique}

La dimension financière est une dimension substantielle à la performance publique et particulièrement clivante. À la fois objectif et contrainte, elle enferme la mise en œuvre de la performance publique dans une impasse lorsqu'elle est insuffisamment pensée. Alors que « la budgétisation est un des éléments les plus politiques dans le processus de décision » (Proulx, Machiavelli, 2005), elle est appréhendée parfois sans nuance. Facette la plus visible de la performance publique, elle est au centre des interrogations dans le cas ici étudié : au-delà du niveau restrictions budgétaires, c'est leur sens et leur cohérence avec les choix de politiques publiques qui sont source de tension (voir encadrés 4 et 5). Le choix de la municipalité est paradoxal : alors que la performance financière est une résultante de choix de gestion, elle a été considérée comme clé d'entrée. Face aux impératifs budgétaires et à l'absence de stratégie stabilisée, les directives de réductions budgétaires ont concerné l'ensemble des directions, sans articulation avec le projet municipal. L'efficacité du procédé est remise en question par l'exécutif et la direction générale. 


\section{Encadré 4 - Expression relative à l'efficience des choix financiers (1/2) (extrait)}

"C'est super frustrant pour un élu de passer son temps à couper $2000 €$ par-ci par-là sans évaluer ce qu'on fait. On le fait de manière comptable et pas politique pour trois ronds.» (Exécutif)

"Les orientations prises sont clairement mathématiques, on n'intègre pas la politique et le service rendu. » (DG)

Non seulement ce mode opératoire est insatisfaisant pour les politiques comme pour l'administration, mais par ailleurs il ne permet pas d'opérer un changement de culture.

\section{Encadré 5 - Expression relative à l'efficience des choix financiers (2/2) (extrait)}

«Élus et directeurs considèrent que quand on supprime quelque chose c'est qu'on considère qu'ils ont mal bossé. Il faut réussir à lever les tabous. Aucune dépense n'est taboue ou sacrée. On doit pouvoir les remettre en cause. » (Exécutif)

"En matière budgétaire, il faut une approche plus globale et priorisée. On reconduit les budgets à l'infini sans réinterroger ce qu'on fait. On demande un peu plus pour avoir un peu moins. Il faut sortir de cette logique. » (Exécutif)

«Les orientations prises sont clairement mathématiques, on n'intègre pas la politique et le service rendu. » (DG)

Si la dimension financière fait partie intégrante d'une performance socialement responsable, elle ne peut la résumer. Or, en l'absence de définition politique et stratégique claire (cf. 3.1.a), élus et administratifs concentrent leur attention sur cet aspect de la performance. En effet, le double caractère structurant de la dimension financière, à la fois tangible, à travers les lignes budgétaires, et récurrent, à travers les débats budgétaires annuels, amène les acteurs à surpondérer les questions financières dans leur approche de la performance. Ceci, au détriment des dimensions dont l'appréhension s'avère plus difficile, faute d'explicitation suffisamment partagée du projet politique et stratégique, et de système de pilotage qui pourrait en découler. Les questions organisationnelles ou celle de l'impact de l'action publique sur les populations ou territoires sont particulièrement concernées. Ce «mauvais » prisme financier, fondé univoquement sur les dépenses et les recettes visibles (budgétées) peut constituer un obstacle important au développement d'une performance socialement responsable.

\section{La gestion des tensions entre politique et administration}

Concernant l'organisation de l'activité, les méthodes de travail sont pointées comme freinant la performance de l'action publique. Plus exactement, c'est la « lourdeur du fonctionnement de l'administration » qui est particulièrement mise en cause par le politique (voir encadré 6). On retrouve là les faiblesses de l'organisation bureaucratique, notamment le prisme de la règle, et la succession de niveau hiérarchique, auxquelles s'ajoutent les défaillances de communication, et par conséquent de synchronisation interne. 


\section{Encadré 6 - Expression relative aux méthodes de travail (extrait)}

«Dans notre collectivité, c'est complètement sclérosé car on ne sort pas de la règle même si elle bloque tout. On se met tellement de contraintes qu'on devient inefficace et cela n'a plus de bon sens. » (Exécutif)

Autre point de cristallisation des tensions, la répartition des rôles entre politique et administratif (voir encadré 7). Cette question est particulièrement soulignée par l'exécutif qui évoque cette problématique à hauteur de $9 \%$ de son expression totale, tandis que les membres de la DG ne l'évoquent qu'à hauteur de $1 \%$. L'exécutif pointe le manque de force de proposition de l'administration et évoque dans le même temps sa tendance à prendre des décisions seule et à mener les affaires en vase clos. Ces difficultés de positionnement sont liées à la répartition des rôles de chacun, mais également à l'équilibre entre concertation et autonomie qui n'est que trop faiblement établi. Au-delà de cet équilibre, l'expression de l'exécutif témoigne de la crainte de se voir déposséder de prérogatives, au bénéfice d'une administration qui s'autonomiserait.

\section{Encadré 7 - Expression relative à la répartition des rôles entre politique et administration (extrait)}

"Il y a plein de gens dans l'administration qui n'admettent pas qu'il y a des élus. Ils font tourner la boutique. Les services font la politique tout seuls, ils n'ont pas besoin de nous. » (Exécutif)

\subsection{La définition de trois axes de travail pour développer la performance durable}

Trois axes de travail issus des séances de groupe de projet menées au sein de la mairie sont particulièrement intéressants dans une perspective de développement coproduit de performance socialement responsable : la reconfiguration plus transversale des processus de décision, la structuration du budget par politiques publiques et non plus nature de dépenses, la mise en place de pratiques d'évaluation des politiques publiques.

Le premier concerne la conduite de l'action publique dans sa dimension politique, à travers la reconfiguration d'une part substantielle du processus de décision. Les neuf pôles de politiques publiques, en place depuis une dizaine d'années, ont été reconfigurés afin de correspondre aux axes structurants du projet de l'équipe municipale, et de favoriser la transversalité entre délégations. Trois pôles de discussions et arbitrages politiques sont désormais en place : cohésion sociale et vivre ensemble, développement du territoire et cadre de vie, politiques éducatives. À travers cette reconfiguration, il s'est agi d'orienter davantage les pôles vers les impacts attendus des politiques publiques, et donc d'amorcer un virage dans le passage de la logique de moyens, à celle de résultats. Cette logique de résultats réinterrogeant la place des gestionnaires publiques face aux élus (Mazouz, 2008; Facal, Mazouz, 2013), une des conséquences directes de cette décision a été la redéfinition de l'équilibre politico-administratif. Le deuxième résultat marquant du groupe de projet est 
celui de la restructuration du budget présenté dorénavant par politiques publiques et non plus par nature de dépenses. L'objectif de la mise en place d'une structuration budgétaire fondée sur l'arborescence des politiques publiques et non plus par nature de dépense, dans l'esprit du cadre fixé par la LOLF pour l'État, est de relier impératif financier et stratégie d'action publique, dans une optique de gestion publique plus performante alliant efficacité et efficience. Ainsi, les discussions budgétaires peuvent servir de base à la structure de l'ensemble de l'action publique, dans la mesure où cette question budgétaire n'est pas appréhendée uniquement à l'aune de l'enveloppe globale à respecter, mais est intégrée au pilotage de l'action publique. Le troisième résultat, fortement lié aux deux précédents, est celui de la mise en place de pratiques d'évaluation des politiques publiques. La mise en place de nouveaux pôles de politiques publiques, ainsi qu'une structuration budgétaire par destination et non par nature, favorisent l'émergence de pratiques d'évaluation. Budget et évaluation peuvent être articulés par le biais d'indicateurs d'évaluation in itinere et intégrée. Ces derniers contribuant au processus de décision, notamment budgétaire, revêtiraient une utilité propice à leur renseignement régulier et effectif. Il ne s'agit donc pas de limiter l'évaluation à une dimension financière ou à la fonction de production interne de l'action publique au sens de Gibert (1980), mais bien d'utiliser cette structuration budgétaire comme base de développement de pratiques intégrées d'évaluation.

\subsection{Discussion des résultats : facteurs génériques versus facteurs contingents de la performance durable d'une collectivité territoriale}

La première phase de la recherche-intervention présentée dans l'article a mis en lumière, en résumé, trois freins au développement de la performance durable de la mairie étudiée : le manque de clarté de la définition politique et stratégique, la surpondération de la dimension financière et enfin les tensions de rôles entre politique et administration. Ces résultats nous semblent rejoindre, formulés sous une forme spécifique, la littérature sur le management public mobilisée dans la première partie de l'article. Leur apport différentiel est de proposer une méthode opératoire de management pour réduire ces freins au travers la co-construction et la coproduction d'actions. Les résultats sont également remarquables en ce qu'ils résultent, non pas d'un œil extérieur - auditeur, consultant, expert etc. - mais d'une expression des acteurs exécutifs et administratifs de la mairie validée de façon collégiale. En ce sens, ils sont la marque du début d'un changement tant il est vrai que le début d'une thérapie de changement consiste en la reconnaissance par les acteurs concernés des dysfonctionnements qui les perturbent (Savall, Zardet, Goter, 2012). Les solutions coproduites en groupe de projet sont quant à elles probablement non duplicables en l'état dans une autre collectivité territoriale. Néanmoins, elles sont en ligne également avec le besoin de synchronisation, de pilotage et de nouveaux modes d'évaluations de la performance publique souligné par la littérature notamment socio-économique. En première analyse certes, ces solutions, d'autant qu'elles n'ont pas été encore complètement mises en œuvre à la fin de la première phase de l'intervention, peuvent sembler modestes. D'autre part, le fait d'avoir décalé dans une seconde phase ultérieure le calcul des coûts cachés - par précaution compte tenu de l'aversion supposée aux coûts des acteurs de la mairie - a retardé l'évaluation économique des réserves endogènes de performances de la mairie et donc la prise de conscience par l'exécutif et l'administratif de la nécessité d'un changement plus rapide. D'un autre coté, ces 
solutions projetées pour modestes soient-elles sont paradoxalement remarquables : jamais la mairie de toute son existence n'avait réalisé un tel exercice de réflexivité collective puis de formalisation politico-administrative collégiale d'axes de changement pour développer sa performance durable. En somme les prémices jamais vues à l'échelle de cette mairie d'une performance publique co-construite et coproduite.

C'est pourquoi, selon nous, les résultats de la recherche doivent donc être analysés sous l'angle de leur contenu et de leur contenant. Au plan de leur contenu, ils sont limités à ce stade. Au plan de leur contenant, ils sont riches de potentialités. Ils confirment en particulier que les outils et les méthodes de mise en œuvre et d'évaluation de la performance publique incitent à rompre avec les modèles top / down classiques de réduction des coûts (Cappelletti, 2012). Ils invitent plutôt le gestionnaire public soucieux de performances durables à s'orienter vers des démarches participatives, négociées, décentralisées et auto-évaluées reposant sur la co-construction et la coproduction d'actions. Cette voie que nous qualifions de socio-économique (Savall, Zardet, Goter, 2012) - peut permettre une meilleure efficience de la production de services publics et de sa mesure sans mutiler l'action publique ni dégrader la satisfaction des clients citoyens et celle des agents producteurs. Elle offrirait ainsi une alternative à la compensation des baisses de dotations publiques par des augmentations de fiscalité que les citoyens usagers ne semblent plus vouloir accepter (Cappelletti, 2016). En grandes lignes, les méthodologies socio-économiques qui permettent ces mouvements féconds de recyclage des coûts en performances pour renouveler la production de services publics, voire la développer de façon autofinancée, reposent sur trois dispositifs essentiels partiellement expérimentés dans la première phase de la rechercheintervention présentée : des diagnostics co-construits et partagés des dysfonctionnements et des coûts qu'ils engendrent, des groupes de projet d'innovation et de coproduction de solutions, un comité de pilotage porteur des souhaits des citoyens et des contraintes publiques de supervision des travaux. Sur ce dernier point, les résultats de la recherche-intervention confirment avec force l'importance du comité de pilotage. C'est en effet cet organe « politique » composé d'élus décideurs (Maire, Adjoints clés) et des personnels de direction clés (DGS) qui détermine les dysfonctionnements prioritaires à traiter à l'issue des diagnostics, qui orientent les travaux de groupes de projet puis entérine les solutions d'amélioration proposées et en pilote la mise en œuvre effective. Cela montre que si ce type de méthodologie socio-économique est participatif et générateur de dialogues professionnels, il est dans le même temps structuré c'est-à-dire placé sous la maîtrise et l'impulsion permanente de la direction politique et administrative de la collectivité considérée. Dans le cas étudié, en l'absence d'un tel comité de pilotage, les résultats n'auraient pas été remarquables, ni modestes, mais quasiment inexistants. Pour ne pas partir dans tous les sens, la co-construction et la coproduction des politiques publiques - donc une certaine horizontalité - demanderaient en même temps l'engagement permanent des organes de direction politique - donc une certaine verticalité.

\section{Conclusion}

Les résultats de la première phase de la recherche-intervention menée au sein d'une mairie de 900 agents d'une commune de 50000 habitants en 2015 et 2016 ont montré, à l'échelle de cette étude de cas, que les freins au développement de la performance socialement responsable résidaient principalement dans un manque de clarté de la définition politique et stratégique, 
la surpondération de la dimension financière des performances et les tensions de rôles entre politique et administration. Les premières solutions projetées et négociées pour lever ces freins résidant quant à elles dans la reconfiguration plus transversale des processus de décision, la structuration du budget par politiques publiques et non plus nature de dépenses et la mise en place de pratiques d'évaluation des politiques publiques. Et cela en s'appuyant sur des dispositifs managériaux favorisant la co-construction et la coproduction d'actions. Ces résultats confirment dans leur versant diagnostic les causes racines principales des difficultés du développement d'une culture de la performance durable dans les collectivités territoriales à savoir : une conception non partagée de la performance publique et qui reste le plus souvent classiquement « comptable », un manque ou des défauts d'évaluation pertinente des politiques publiques menées et de leurs déclinaisons en actions par les services, une distance cognitive trop importante entre les sphères politique et administrative. Dans leur versant projet et plus globalement, au-delà des solutions projetées au sein de la mairie, les premiers résultats de la recherche invitent à expérimenter au sein des collectivités territoriales des méthodologies de gestion qui au plan conceptuel sont fondées non pas sur un rétrécissement des services publics par la réduction unilatérale des coûts visibles mais sur leur renouvellement par une dialectique de recyclage des coûts en performances. Ces méthodologies d'inspiration socio-économique appellent au plan pratique des outils et des dispositifs décentralisés qui vont stimuler l'implication des agents et des élus et s'intéresser par la négociation à leur satisfaction ainsi qu'à celle symétriquement des usagers citoyens. L'hypothèse fondamentale sous jacente étant que les outils de mesure et de mise en œuvre des politiques publiques visant à améliorer l'efficience ne peuvent se déployer sans et/ ou contre les agents publics concernés ni bien entendu les élus mais demandent au contraire leur coproduction. Peut-être plus encore que dans le secteur privé, la mesure et l'évaluation des performances dans le secteur public nécessite une gestion interactive, intersubjective, décentralisée et synchronisée fondée sur des représentations partagées de la performance et, au fond, la co-construction et la coproduction de la mise en œuvre des politiques publiques soit une certaine horizontalité des processus de gestion. Mais en même, cette horizontalité ne semble pouvoir se déployer en cohérence qu'avec une verticalité affirmée, c'est-à-dire l'engagement permanent des directions administratives et politiques considérées.

En termes de perspectives, la présente recherche-intervention devra bien entendu être analysée à l'aune des résultats de sa deuxième phase prévus pour fin 2017. En particulier la mesure économique des coûts cachés des dysfonctionnements de la mairie, celle des performances créées au travers leur réduction et celle de la satisfaction des parties prenantes de la mairie - agents, élus, usagers-citoyens etc. - pour valider le développement d'une performance socialement responsable. Au-delà de cette étude de cas et des validations analytiques qui ont pu être faîtes au regard de la littérature existante sur la question, d'autres cas d'étude devront être accumulés à l'avenir pour renforcer la robustesse des connaissances proposées, les approfondir et en tracer les limites. Parmi elles, la dialectique horizontalité verticalité comme ressort de la co-construction / coproduction des politiques publiques semble particulièrement riche d'enjeux. 


\section{Bibliographie}

Actes des assises du service public 2015 de la DFCG (Association des directeurs financiers et du contrôle de gestion), Paris, Maison des Polytechniciens, avril.

Actes du congrès 2012 de l'AIRMAP (Association internationale de recherche en management public), Paris 2 - Assas.

Antunes D., 2004. The Transformative Approach In Buono A.F. (ed.), Creative Consulting. Innovative Perspectives on Management Consulting, Information Age Publishing, Charlotte, 303-324.

Argyris C. and Schön D.A., 1978. Organizational Learning. A Theory of Action Perspective, Addison-Wesley, New York.

Argyris C., Putnam R., McLain Smith D., 1985. Action Science. Jossey-Bass, San Fransisco.

Ashworth R., Ferlie E., Hammerschid G., Moon J. and Reay T., 2013. Theorizing Contemporary Public Management: International and Comparative Perspectives. British Journal of Management 241-17.

Bartoli A. et Blatrix C., 2015. Le management dans les organisations publiques. Sens et logiques d'action. Dunod, Paris.

Bartoli A., Kéramidas O., Larat F. et Mazouz B., 2011. Vers un management public éthique et performant. Revue française d'administration publique 140, 629-639.

Boltanski L. et Thevenot L., 1991. De la justification. Les économies de la grandeur. Gallimard, Paris.

Bouckaert G., 2005. Un nouvel examen de la mesure de la performance dans le secteur public. Télescope Revue d'analyse comparée en administration publique 12 (3), 12-25.

Bouckaert G. et Pollitt C., 2004. Evaluating Public Management Reforms: a Comparative Analysis. Oxford University Press, Oxford.

Bouckaert G. and Van Dooren W., 2002. Performance Measurement: Getting Results. Public Performance \& Management Review 25 (3), 329-335.

Buono A. and Savall H. (Eds), 2007. Socio-Economic Intervention in Organizations. The Intervener-Researcher and the Seam Approach to Organizational Analysis. Information Age Publishing, Charlotte.

Burell G. And Morgan G., 1985. Sociological Paradigms and Organizational Analyses. Gower, Aldershot.

Burlaud A. et Chatelain-Ponroy S., 2013. Contrôle de gestion et performances dans le secteur public In Cappelletti, L., Hoarau, C. (dir.), Finance et contrôle au quotidien, Dunod, Paris.

Cabinet Alma Consulting Group, 2015. Enquête 2014 sur l'absentéisme. lepoint.fr, 10 septembre.

Cappelletti L., 2007. L'exploitation des pratiques professionnelles dans une recherche en audit et contrôle : apports de la recherche-intervention qualimétrique. Revue Sciences de Gestion-Management SciencesCiencias de Gestion 59, 67-90.

Cappelletti L., 2012. Le contrôle de gestion de l'immatériel. Une nouvelle approche du capital humain. Dunod, Paris.

Cappelletti L., 2016. Collectivités locales : des pistes pour faire mieux avec moins. Les Échos, 4 janvier, 10.

Carassus D., Favoreu C., Gardey D. et Maurel C., 2011. La caractérisation et la définition de la performance publique : une application aux collectivités locales. Premier colloque AIRMAP, Versailles-Saint Quentin, 29 juin au $1^{\mathrm{er}}$ juillet.

Challenge (2015). «Dossier Fonctionnaires: la France incapable de gérer ses sureffectifs », n 443 du 3 au 9 septembre, pp. 50 à 61 .

Chevallier J. et Loschak D., 1982. Rationalité juridique et rationalité managériale dans l'administration française. Revue française d'administration publique 24, 67 à 90.

Christensen T. and Laegreid P., 2010. Complexity and Hybrid Public Administration - Theorical and Empirical Challenges. Public Organization Review 11 (4), 1-17.

Coghlan D. and Brannick T., 2005. Doing Action Research in Your Own Organization. Sage Publications, London. 
David A., 1999. Intervention methodologies in management research. Track: Collaborative management / Research approach.

David A., 2003. Études de cas et généralisation scientifique en sciences de gestion. Revue Sciences de Gestion $39,139-166$.

David A., 2004. Les connaissances en sciences de gestion: devons-nous choisir entre scientificité et actionnabilité? Traversée des frontières entre méthodes de recherche qualitatives et quantitatives. Acte du colloque AOM-IAE de Lyon, 845-870.

Davis P. and West K., 2009. What Do Public Values Mean for Public Action? Putting Public Values in their Plural Place. The American Review of Public Administration 39 (6), 602-618.

Den Hartog D.N., Boselie P. and Paauwe J., 2004. Performance Management: a Model Research Agenda. Applied Psychology 53 (4), 556569.

Dupuy F., 1998. Le client et le bureaucrate. Dunod, Paris.

Emery Y., 2005. La gestion par les résultats dans les organisations publiques : de l'idée aux défis de la réalisation. Télescope Revue d'analyse comparée en administration publique 12 (3), 1-11.

Favoreu C., Carassus D., Gardey D. et Maurel C., 2015. Le management par la performance dans le secteur public local français : un modèle plus administratif que politique. Revue Internationale des Sciences Administratives 81, 713-734.

Ferlie E., Hartley J. and Martin S., 2003. Changing public service organizations: current perspectives and future prospects. British Journal of Management 14, S1-S14.

Harvard Business Review, 2015. It's Time to Blow Up HR and Build Something new Here's now. July-August, 54-78.

Hatchuel A., Masson P.L. and Weil B., 2002. From Knowledge Management to Design-Oriented Organizations. International Social Science Journal 171, 25-37.

Hudon P.-A. et Mazouz B. (2014). Le management public entre «tensions de gouvernance publique » et « obligation de résultats » : vers une explication de la pluralité du management. Gestion et management public 3 (2), 7-22.

Jönsson S. and Lukka K., 2005. Doing interventionist research in management accounting. Gothenburg Research Institute-rapport 6.

Kaplan R.S., 1998. Innovation Action Research: Creating new management theory and practices. Journal of Management Accounting Research 10, 89-113.

Laufer R. et Burlaud A., 1980. Management public : gestion et légitimité. Dalloz, Paris.

Lee T.W., 1999. Using Qualitative Methods in Organizational Research. Organizational Research Methods. Sage Publications, Thousand Oaks.

Martin V. and Jobin M.-H., 2004. La gestion axée sur les résultats : comparaison des cadres de gestion de huit juridictions. Administration publique du Canada 47 (3), 304-331.

Martinet A.-C., 1991. Management en temps réel et continuité stratégique sont-ils compatibles ? Revue Française de Gestion 86, 52-56.

Maurel C., Carassus D. et Gardey D., 2011. Les démarches locales de performance publique face à la LOLF : mimétisme ou innovation? Politique et Management public 28 (4), 417-442.

Maxwell J.A., 1996. Qualitative Research Design. Sage, Thousand Oaks.

Mazouz B., 2008. Le métier de gestionnaire public à l'aube de la gestion par résultats : nouveaux rôles nouvelles fonctions - nouveaux profils. Presses de l'Université du Québec, Québec.

Mazouz B. et Tardif M. J. B., 2010. À propos de la performance : l'Arlésienne de la sphère publique In D. Proulx, Management des organisations publiques, Québec, Presses de l’Université du Québec, Québec.

Mazouz B. et Tremblay B., 2006. oward a Postbureaucratic Model of Governance: How Institutional Commitment Is Challenging Quebec's Administration. Public Administration Review 66 (2), 263-273.

MazouzB., Leclerc J. et Tardif M.J.-B., 2008. La gestion intégrée par résultats : concevoir et gérer autrement la performance dans l'administration publique. Presses de 1'Université du Québec, Québec. 
Mazouz B., Sponem S. et Rousseau A., 2015. Le gestionnaire public en question. La difficile conciliation des logiques bureaucratique et managériale. Revue française de gestion 250, 89-104.

Mckelvey B., 2006. Van de Ven and Johnson's Engaged Scholarship: Nice Try, but... The Academy of Management Review 31 (4), 822-829.

McKernan J., 1991. Curriculum Action Research. A Handbook of Methods and Resources for the Reflexive Practitioner. Kogan Page, London.

Meier K., Andersen S.C., O’Toole Jr. L.J., Favero N. and Winter S., 2015. Taking managerial context seriously: public management and performance in US and Denmark schools. International Public Management Journal 18 (1), 130-150.

Meyer R.E., Egger-Peitler I., Höllerer M.A. et Hammerschmid G., 2014. Of Bureaucrats and Passionate Public Managers: Institutional Logics, Executive Identities, and Public Service Motivation. Public Administration 92 (4), 861-885.

Moynihan D. P., 2006. Managing for Results in State Government: Evaluating a Decade of Reform. Public Administration Review 66 (1), 7890.

Moynihan D.P., 2008. The Dynamics of Performance Management : Constructing Information and Reform. Georgetown University Press, Washington DC.

Noguera F., 2006, 2008. «Diagnostic et projet d'amélioration des outils de contractualisation d'un conseil régional » et « Diagnostic et projet d'amélioration d'un centre régional des impôts ». Rapport de rechercheintervention ISEOR-Magellan.

Noguera F., 2010. Les défis posés par la nouvelle gestion publique à la GRH : cas de la Direction Générale des impôts. Revue Gestion des Ressources Humaines 78, 32-58.

Nooteboom B.; 2000. Learning by interaction: absorptive capacity, cognitive distance and governance. Journal of Management and Governance 4, 69-92.

Plane J.M., 2000. Méthodes de recherche-intervention en management. 1'Harmattan, Paris.

Proulx D. et Machiavelli F., 2005. La conception de la gestion par résultats en Amérique latine. Télescope Revue d'analyse comparée en administration publique 12 (3), $42-53$.

Revue Économie et Management, 2015. «Dossier enjeux et dimensions du management public » 154.

Ring P. and Perry J.L., 1985. Strategic Management in Public and Private Organizations: Implications of Distinctive Contexts and Constraints. Academy of Management Review 10 (2), 276-286.

Robert C., 2007. Les transformations managériales des activités politiques. Politix 79, 7-23.

Robin J-Y. et Desfontaines H., 2015. Le DGS, fonctionnaire manager et entrepreneur politique? 13e congrès national de l'AFSP, Aix-en-provence.

Savall H. et Zardet V., 2010. La métamorphose des services publics : résultats qualimétriques d'amélioration de l'efficience, de l'efficacité et de la qualité des services aux usagers. II Congrès Transatlantique d'audit, comptabilité, contrôle \& gestion des coûts, Instituto Internacional de Costos-American Accounting Association (MAS)-ISEOR, Lyon, Juin.

Savall H., Zardet V. et Bonnet M., 2008. Libérer les performances cachées par un management socio-économique. Éditions B.I.T - O.I.T, Genève.

Savall H., Zardet V. et Goter F., 2012. Modernisation des services publics : état des lieux et enjeux d'une gestion socio-économique des équipes et des missions. Organizational Development \& Change Division Meeting, Academy Of Management-ISEOR, Lyon, Juin.

Simon H., 1957. Models of Man: Social and Rational. Mathematical Essays on Rational Behavior in a Social Setting. Wiley, New York.

Trosa S., 1989. Grandeur et décadence du mimétisme avec le secteur privé. Politique et management public 7 (2), 237-250.

Van de Ven A.H. and Johnson P.E., 2006. Knowledge for Theory and Practice. The Academy of Management Review 31 (4), 802-821. 
Van Hée N., 2008. Distance cognitive et capacités d'absorption : deux notions étroitement imbriquées dans les processus d'apprentissage et d'innovation. Revue d'économie industrielle 121, 103-124.

Wacheux F., 1996. Méthodes qualitatives et recherche en gestion. Economica, Paris.

Zardet V. et Noguera F., 2013. Quelle contribution du management au développement de la dynamique territoriale ? Expérimentation d'outils de contractualisation sur trois territoires. Revue Gestion et Management Public 2 (2), 5-31. 
